\title{
Is Hydrogen Peroxide Disinfection Effective for Cleaning Pollen Particles Attached to Contact Lenses?
}

This article was published in the following Dove Press journal: Clinical Optometry

\author{
Takahiro Sunaga ${ }^{1,2}$ \\ Tatsuya Mimura' \\ Hiroshi Matsuoka ${ }^{2}$ \\ Hiroaki Horikawa' \\ Kazuma Kitsu (D) \\ Atsushi Mizota'
}

'Department of Ophthalmology, Teikyo University School of Medicine, Itabashiku, Tokyo, Japan; ${ }^{2}$ Teikyo University of Science \& Technology, Senju Campus 7, Adachi-ku, Tokyo, Japan
Correspondence: Tatsuya Mimura

Tel +8I-3-3964-|2|I

Fax +8I-3-3964-I 1402

Email mimurat-tky@umin.ac.jp
Purpose: It has been proven that wearing soft contact lenses (SCLs) can make polleninduced allergic conjunctivitis worse. We investigated the cleaning effect of disinfectants and rinsing solution on cedar pollen attached to SCLs.

Methods: Two-week replacement disposable SCLs, to which pollen particles were experimentally attached, were washed and cleaned with either saline of rinsing solution $(n=10)$ or $3 \%$ hydrogen peroxide $(n=10)$. We observed, under a microscope, the cedar pollen adhered to the SCLs after washing.

Results: The number of residual pollens attached to SCLs was $23.7 \pm 25.7$ with rinsing solution and $0.2 \pm 0.6$ with $3 \%$ hydrogen peroxide solution per single area $(100 \mu \mathrm{m} \times 100$ $\mu \mathrm{m})$. The percentage of adhesion area of pollen to the SCLs was $0.9 \pm 1.1 \%$ with rinsing solution and $0.0 \pm 0.0 \%$ with $3 \%$ hydrogen peroxide solution. There were significant differences in the number of pollen and adhesion areas of pollen between the two groups ( $\mathrm{p}<0.05$ and $\mathrm{p}<0.05$, respectively).

Conclusion: These results suggest that hydrogen peroxide solution is superior in cleaning effect of cedar pollen attached to SCLs compared to saline.

Keywords: hydrogen peroxide, pollen, disinfection, soft contact lens

\section{Introduction}

Seasonal allergic conjunctivitis is eye inflammation induced by an allergic reaction to substances, such as pollens and molds. ${ }^{1,2}$ Therefore, seasonal allergic conjunctivitis worsens during the same season every year. ${ }^{1,2}$ In addition, allergic conjunctivitis is exacerbated by foreign body reaction caused by wearing a soft contact lens (SCL) during the hay fever season. The moist surfaces of SCLs may also trap various allergens, such as pollens, and cause worse symptoms of allergic conjunctivitis. Thus, daily cleaning and disinfection of SCLs are required when using conventional SCLs or frequent-replacement SCLs to remove pollens and protein components attached to the SCLs for self-care on the patient's side.

SCL disinfectant solutions include povidone-iodine, multi-purpose solutions (MPS) using chemical disinfectants, and hydrogen peroxide-based solutions. Hydrogen peroxide has been reported to be superior to MPS in disinfecting and cleaning effects. $^{1-5}$ It has also been reported that microorganisms such as bacteria, viruses, fungi, and Acanthamoeba attached to SCLs are killed by soaking with 3\% hydrogen peroxide for 6 hours. ${ }^{6}$ However, the cleaning effect of hydrogen peroxide on 
pollens attached to SCLs is not well understood. We examined the cleaning effect of commercially available hydrogen peroxide solutions on pollen components adhering to SCLs.

\section{Methods}

\section{Research Design and Test Chemicals}

This study was a randomized and non-clinical study. This study was approved by the Ethics Committee of Teikyo University as a study on the causative bacteria of ocular infections, including contact lens contamination (\#Teirin 18-227) and performed at an ophthalmology laboratory of Teikyo University School of Medicine from April 2019 to September 2019. Hydrogen peroxide solutions (AOSept Clear Care, Alcon Japan, Minato-ku, Tokyo, Japan) and physiological saline (Otsuka Pharmaceutical Co., Ltd) were purchased. Details of the drug solutions used in this test are shown in Table 1. The sample size was ten in the group using physiological saline (saline group) and ten in the group using hydrogen peroxide solution (hydrogen peroxide group).

\section{Cleaning Method of SCL}

Japanese cedar pollen collected on March 3, 2019, was used in this study (Yamizo Pollen Study Group, Daigo-cho, Ibaraki Prefecture, Japan). A drop of $0.2 \mathrm{~mL}$ of physiological saline containing $0.01 \mathrm{mg} / \mathrm{mL}$ of cedar pollen was dropped inside unused SCLs (J \& J 2 week Acuvue; Johnson \& Johnson, Chiyoda-ku, Tokyo, Japan) and kept at room temperature for 1 hour. The details of SCL are as follows: FDA group (IV), principal components (2-HEMA, MAA), USAN

Table I Details and Composition of Cleaning Solution

\begin{tabular}{|c|c|}
\hline \multicolumn{2}{|c|}{ Physiological Saline } \\
\hline Chemical & Otsuka Normal Saline \\
\hline Name & \\
\hline Active & None \\
\hline Ingredient & \\
\hline Ingredients & Sodium chloride $0.18 \mathrm{mg} / 20 \mathrm{~mL}$ \\
\hline Manufacturer & Otsuka Pharmaceutical Co., Ltd. \\
\hline \multicolumn{2}{|c|}{ Hydrogen Peroxide Solution } \\
\hline Chemical & AOSept Clear Care \\
\hline Name & \\
\hline Active & Hydrogen peroxide $3.42 \mathrm{w} / \mathrm{v} \%$ \\
\hline Ingredient & \\
\hline Ingredients & Stabilizers, Buffers, pH adjusters, Tonicity agents, \\
\hline & Surfactants (Polyoxyethyleneoxypropylene glycol) \\
\hline Manufacturer & Alcon Japan Ltd. \\
\hline
\end{tabular}

nomenclature (etafilcon A), water content (58\%), oxygen permeability (DK; $28.0 \times 10^{-11}(\mathrm{~cm} 2 / \mathrm{sec})(\mathrm{mlO} 2 / \mathrm{mL} \times \mathrm{mmHg})$ ), and oxygen transmissibility $\left(\mathrm{Dk} / \mathrm{L} ; 33.3 \times 10^{-9}(\mathrm{~cm} \times \mathrm{mLO} 2 /\right.$ sec $\times \mathrm{mL} \times \mathrm{mmHg}$ ). After 1 hour, the SCLs were randomly divided into two groups, the saline group $(n=10)$ and the hydrogen peroxide group $(\mathrm{n}=10)$, and then the SCLs were rubbed and rinsed three times. The rubbing method was as follows: the lens was placed on the palm, a few drops of physiological saline were applied, and both sides of the lens were rubbed lightly with fingers 30 times. The SCLs were further rinsed with physiological saline. The SCLs were placed in the lens holder of a disposable cup and were soaked with each chemical solution for 6 hours at room temperature according to the manufacturer's instructions. The SCLs were further rinsed three times with physiological saline. The central part of the inner surface of the SCL was observed under a microscope (BX-61, Olympus, Tokyo, Japan) attached with a CCD camera. And the images were first captured in high TIFF format with an original size of 4800x3600 pixels (width $\times$ height) in 24-bit red, green and blue (RGB) color at 72 pixels per inch resolution and transferred to a computer equipped with the image analysis software to be analyzed. Additionally, the observed images were converted to the JPEG format for the analysis. The number of particles adhering to the area of $100 \mu \mathrm{m} \times 100 \mu \mathrm{m}$ in the central part of the saved image (the central part of CL) and the ratio of the adhering area were analyzed using ImageJ analysis software (version 1.52a; Wayne Rasband, NIH, Bethesda, MD, http://imagej.nih.gov/ ij/docs/index. html).

\section{Method of Randomization and Statistical Analysis}

Numbers were sequentially assigned to the lenses, and a cleaning solution was determined for each lens using a random number table. The measurements were performed in the order of numbers. An independent sample $t$-test was used for comparison between the two groups, saline and hydrogen peroxide.

\section{Results}

\section{Pollen Particles Adhered to SCL Before Washing}

Figure 1 shows photographs of the pollen particles after being left on the SCLs for 1 hour and after being washed with physiological saline or hydrogen peroxide solution. Figure 2 shows the number of pollen particles and the adhesion area immediately after being left on the SCLs for 1 hour. There 


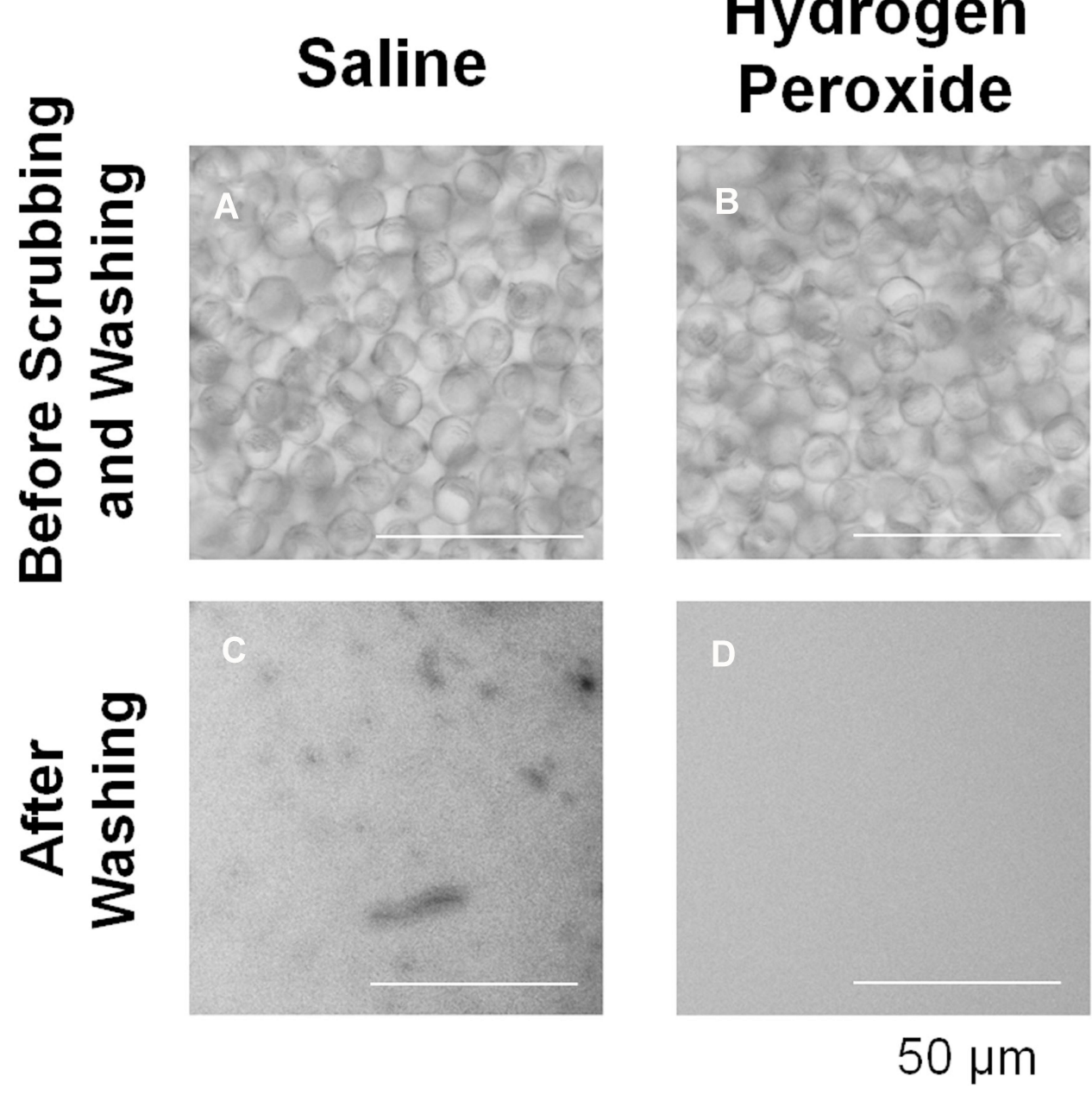

Figure I Photographs of pollen particles attached to 2-week replacement soft contact lenses (SCL). (A and B) Photographs of pollen adhered to the inner surface of an SCL for I hour. (C and D) Photograph of pollen particles remaining on SCL after washing with physiological saline or hydrogen peroxide solution. Bars $=50 \mu \mathrm{m}$.

was no difference in the number of pollen particles $(p=$ 0.1537 , Figure $2 A)$ and the area of adhesion $(p=0.7473$, Figure 2B) between the saline and hydrogen peroxide group.

\section{Pollen Particles Adhered to SCL After Washing with a Washing Solution}

Figure 3 shows photographs of pollen particles after washing with physiological saline $(\mathrm{n}=10)$ or hydrogen peroxide $(n=10)$ for 6 hours. The number of pollen particles attached to the SCL per single area $(100 \mu \mathrm{m} \times 100 \mu \mathrm{m})$ was $23.7 \pm 25.7$ in the saline group and $0.2 \pm 0.6 / 100 \mu \mathrm{m} \times$ $100 \mu \mathrm{m}$ in the hydrogen peroxide group (Figure 4A). There was a significant difference between the two groups (independent sample $t$-test, $\mathrm{p}=0.0134$ ).

Figure 4B shows the ratio (occupancy rate) of the adhesion area of pollen particles. The percentage of pollen adhered area was $0.9 \pm 1.1 \%$ in the saline group and $0.0 \pm$
$0.0 \%$ in the hydrogen peroxide group. There was a significant difference between the two groups (independent sample $t$-test, $\mathrm{p}=0.0321)$.

\section{Discussion}

To date, there are many studies on the antibiotic effect of hydrogen peroxide against microorganisms attached to SCLs. ${ }^{1-7}$ For example, hydrogen peroxide was effective against clinical bacterial isolates and not affected by organic soils in tears. Retuerto and associates examined microbiome adherent to CLs and the association of bacterial communities with the use of lens care solutions. They reported that Corynebacterium, Haemophilus, and Streptococcus were more detected in the MPS-treated CLs compared with hydrogen peroxide-treated CLs. ${ }^{9}$ Guillon and associates also examined the effect of two lens care systems on silicone hydrogel contact lens wettability. They reported that contact 


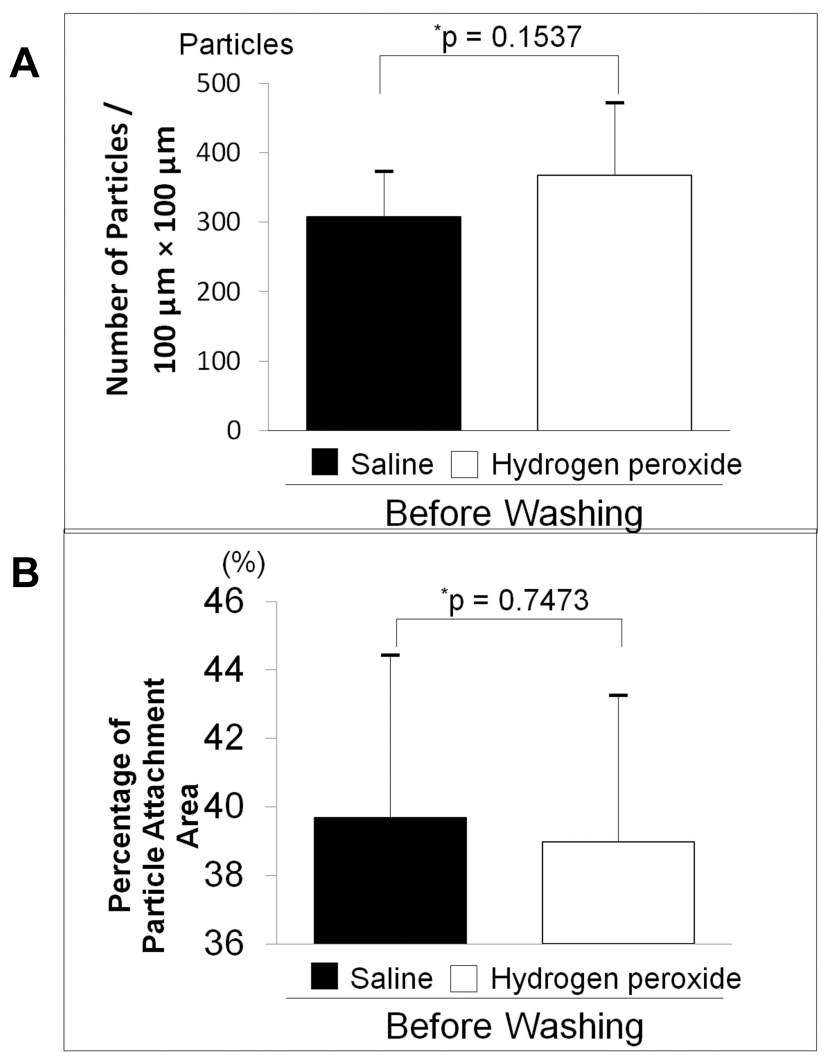

Figure 2 Comparison of pollen particles adhered to soft contact lenses (SCL) after scrubbing with physiological saline and hydrogen peroxide. (A) Number of pollen particles attached per single area $(100 \mu \mathrm{m} \times 100 \mu \mathrm{m})$ in the central part of the SCL. (B) Percentage of adhesion area of pollen particles on the inner surface of the $\mathrm{SCL}$ (\%). *P value: Independent sample $t$-test was used for comparison between the two groups. Values are expressed as mean \pm standard deviation.

lens wettability and surface cleanliness were significantly better in hydrogen peroxide cleaning and disinfecting system than in MPS at the end of three months of use. ${ }^{10}$ These results suggest that hydrogen peroxide is superior to MPS in disinfecting and cleaning effects. However, there are few studies on the cleaning effect of pollen attached to SCLs by disinfectants. This is the first study to verify the effect of cleaning pollen attached to SCLs by hydrogen peroxide solution.

In this study, hydrogen peroxide-based contact lens cleaning was superior to physiological saline in cleaning effect on pollen particles adhering to SCLs. The hydrogen peroxide solution is also used as a general disinfectant, oxidol, and is also used for disinfecting wounds and sanitary equipment. Hydrogen peroxide is rapidly decomposed into oxygen during disinfection, and its half-life in vivo is extremely short. Therefore, hydrogen peroxide itself disappears after the disinfection of the SCL. Catalase, a hydrogen peroxide-degrading enzyme, is widely distributed in many plants, animals, and microorganisms except for anaerobic bacteria. ${ }^{11}$ Hydrogen peroxide $\left(\mathrm{H}_{2} \mathrm{O}_{2}\right)$ is converted into oxygen and water in vivo by a disproportionation reaction by catalase and subsequently eliminated. A large amount of oxygen is generated in the process of hydrogen peroxide water being decomposed by the reaction with catalase. Therefore, it is possible that large amounts of oxygen bubbling occurs while decomposing and removing pollen particles from SCLs. These results show that hydrogen peroxide disinfection not only has the effect of disinfecting microorganisms ${ }^{12,13,14}$ but also of physically removing proteins and foreign substances by foaming oxygen generated by decomposition from the hydrogen peroxide solution. Additionally, we previously confirmed that 3\% hydrogen peroxide burst pollen independent of contact lenses. ${ }^{15}$ Our previous study also showed that saline can cause the pollen

\section{Saline}

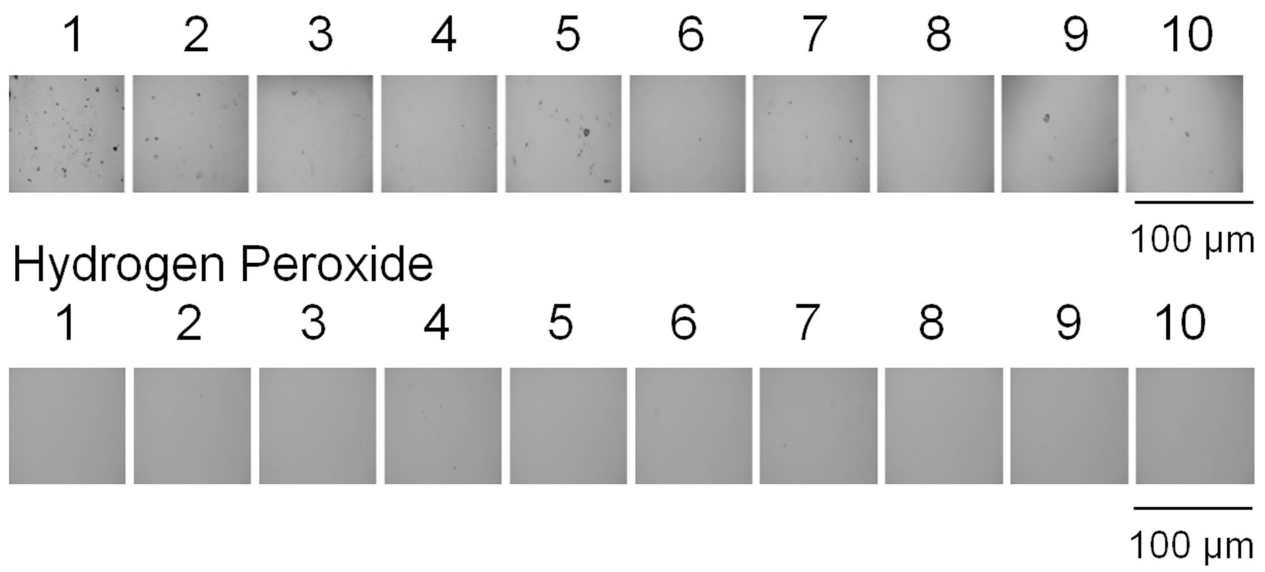

Figure 3 Photographs of pollen particles attached to the inner surface of a soft contact lens $(\mathrm{SCL})$ after washing with physiological saline or hydrogen peroxide. Bars $=100$ $\mu \mathrm{m}$. 


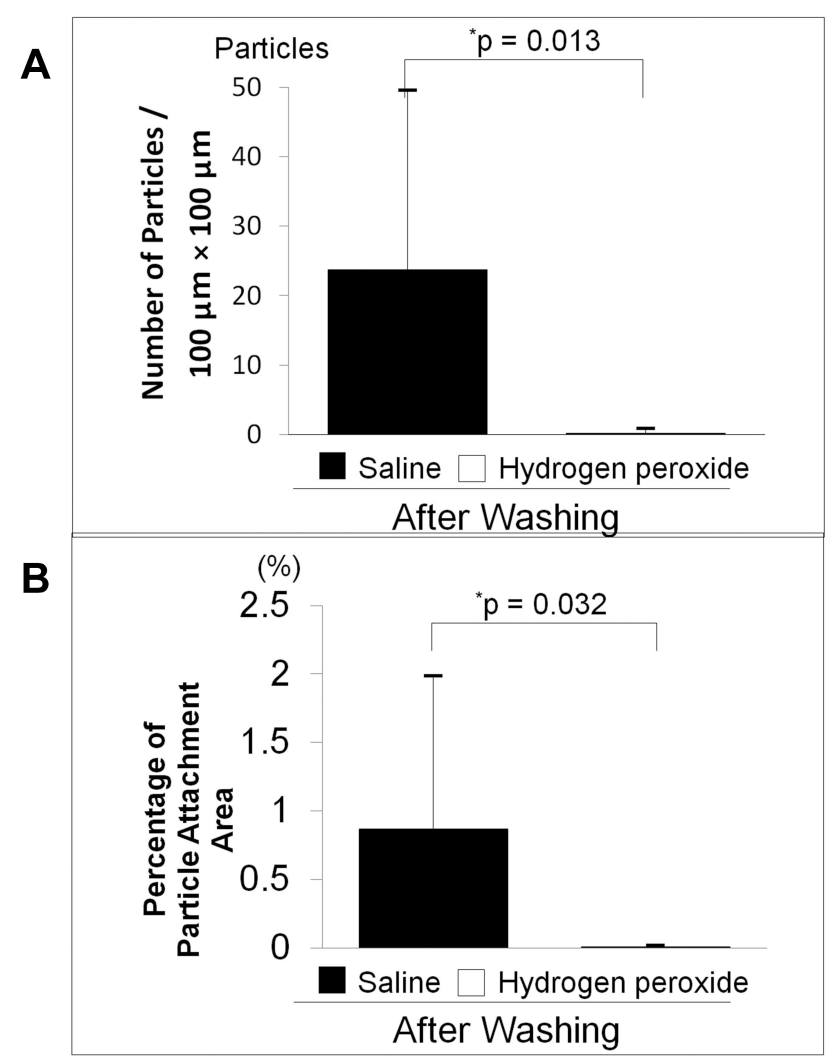

Figure 4 Comparison of pollen particles adhered to soft contact lenses $(\mathrm{SCL})$ after washing with physiological saline and hydrogen peroxide. (A) Number of pollen particles attached per single area $(100 \mu \mathrm{m} \times 100 \mu \mathrm{m})$ in the central part of the SCL. (B) Percentage of adhesion area of pollen particles on the inner surface of the $\mathrm{SCL}$ (\%). *P value: Independent sample $t$-test was used for comparison between the two groups. Values are expressed as mean \pm standard deviation.

to burst by osmotic pressure and the burst rate was higher with 24-hour exposure than with 1-hour exposure. Oxygen bubbles generated from hydrogen peroxide could cause pollen to burst more than water or saline. ${ }^{15}$

Zhu and associates compared three types of SCL cleaning techniques in the use of MPS, including 1) rub and rinse before soaking, 2) rinse-only: no rubbing step, and 3) no rub and no rinse: soaking only. ${ }^{16}$ They concluded that 'rub and rinse' is the most effective regimen to disinfect microorganisms remaining on SCLs. ${ }^{16}$ Our previous study also demonstrated that the pollen particles remained attached to the surface of SCLs after rinsing 3 times but most of the pollen were removed by rubbing the SCL strongly with the fingers. ${ }^{15}$ Therefore, the best way of cleaning contact lenses may be rub and rinse.

This study has some limitations. First, the amount of pollen used was not realistic, and the study was conducted under rather severe conditions. The general airborne amount of pollen is much less than the experimental amount. Second, this study only compared hydrogen peroxide disinfection with physiological saline although MPS is the common choice as disinfectants. Previously, we compared the disinfecting effects of hydrogen peroxide disinfection with MPS in a 24-hour long-term exposure experiment of pollen particles to SCLs. ${ }^{15}$ In the future, it is necessary to examine the comparison between hydrogen peroxide solution and other disinfectants. Third, it is difficult to verify the true effect of hydrogen peroxide solution itself because the commercial solution contains other ingredients. However, the ingredients including surfactants of polyoxyethyleneoxypropylene glycol are used as an emulsifier for solutions and have no sterilizing effect on organisms. Therefore, we think that this study has adequately demonstrated the pollen cleaning effect of commercial hydrogen peroxide solutions.

\section{Conclusion}

Various particles such as proteins, foreign substances, and pollen attached to SCLs may exacerbate allergic conjunctivitis. This study demonstrated that AOSept Clear Care with hydrogen peroxide has an excellent effect of removing pollen components adhering to 2-week replacement disposable type SCLs.

\section{Funding}

This work was supported in part by a Grant-in-Aid for Scientific Research from the Ministry of Education, Culture, Sports, Science and Technology of Japan [grant number 20H04347] and an unrestricted investigatorinitiated grant from Alcon Japan Ltd. to Tatsuya Mimura, MD. The funders had no role in study design, data collection and analysis, decision to publish, or preparation of the manuscript.

\section{Disclosure}

No author has a financial or proprietary interest in any material or method mentioned. The authors report no conflicts of interest in this work.

\section{References}

1. Mimura T, Amano S, Funatsu H, et al. Correlations between each allergen-specific IgE serum levels in patients with allergic conjunctivitis in spring. Ocul Immunol Inflamm. 2004;12(1):45-51. doi:10.1076/ocii.12.1.45.28067

2. Mimura T, Yamagami S, Amano S, et al. Allergens in Japanese patients with allergic conjunctivitis in autumn. Eye. 2005;19 (9):995-999. doi:10.1038/sj.eye.6701701

3. Kobayashi T, Gibbon L, Mito T, et al. Efficacy of commercial soft contact lens disinfectant solutions against Acanthamoeba. Jpn J Ophthalmol. 2011;55(5):547-557. doi:10.1007/s10384-011-0062-y 
4. Lorentz H, Heynen M, Tran H, et al. Using an in vitro model of lipid deposition to assess the efficiency of hydrogen peroxide solutions to remove lipid from various contact lens materials. Curr Eye Res. 2012;37(9):777-786. doi:10.3109/02713683.2012.682636

5. Gabriel MM, McAnally C, Bartell J, et al. Biocidal efficacy of a hydrogen peroxide lens care solution incorporating a novel wetting agent. Eye Contact Lens. 2019;45(3):164-170. doi:10.1097/ICL. 0000000000000549

6. Kilvington S, Winterton L. Fibrous catalyst-enhanced acanthamoeba disinfection by hydrogen peroxide. Optom Vis Sci. 2017;94 (11):1022-1028. doi:10.1097/OPX.0000000000001126

7. Nichols JJ, Chalmers RL, Dumbleton K, et al. The case for using hydrogen peroxide contact lens care solutions: a review. Eye Contact Lens. 2019;45(2):69-82. doi:10.1097/ICL.0000000000000542

8. Yamasaki K, Mizuno Y, Kitamura Y, et al. The antimicrobial activity of multipurpose disinfecting solutions in the presence of different organic soils. Eye Contact Lens. 2020;46(4):201-207. doi:10.1097/ ICL.0000000000000694

9. Retuerto MA, Szczotka-Flynn L, Mukherjee PK, et al. Diversity of ocular surface bacterial microbiome adherent to worn contact lenses and bacterial communities associated with care solution use. Eye Contact Lens. 2019;45(5):331-339. doi:10.1097/ICL.0000000000000 578
10. Guillon M, Maissa C, Wong S, Patel T, Garofalo R. Effect of lens care system on silicone hydrogel contact lens wettability. Cont Lens Anterior Eye. 2015;38(6):435-441. doi:10.1016/j.clae.2015.06.007

11. Brioukhanov AL, Netrusov AI, Eggen RIL. The catalase and superoxide dismutase genes are transcriptionally up-regulated upon oxidative stress in the strictly anaerobic archaeon Methanosarcina barkeri. Microbiology. 2006;152(6):1671-1677. doi:10.1099/mic.0.28542-0

12. Joslin CE, Tu EY, Shoff ME, et al. The association of contact lens solution use and Acanthamoeba keratitis. Am J Ophthalmol. 2007;144(2):169-180. doi:10.1016/j.ajo.2007.05.029

13. Szczotka-Flynn LB, Imamura Y, Chandra J, et al. Increased resistance of contact lens-related bacterial biofilms to antimicrobial activity of soft contact lens care solutions. Cornea. 2009;28(8):918-926. doi:10.1097/ICO.0b013e3181a81835

14. Dannelly HK, Waworuntu RV. Effectiveness of contact lens disinfectants after lens storage. Eye Contact Lens. 2004;30(3):163-165. doi:10.1097/01.icl.0000133562.84179.5d

15. Mimura T, Sunaga T, Mizota A. Clinical academic topics. Cleaning effect of hydrogen peroxide solution on cedar pollen attached to contact lens. (Japanese) Rinsho Allergy. 2020;40(3):70-79.

16. Zhu H, Bandara MB, Vijay AK, et al. Importance of rub and rinse in use of multipurpose contact lens solution. Optom Vis Sci. 2011;88 (8):967-972. doi:10.1097/OPX.0b013e31821bf976
Clinical Optometry

\section{Publish your work in this journal}

Clinical Optometry is an international, peer-reviewed, open access journal publishing original research, basic science, clinical and epidemiological studies, reviews and evaluations on clinical optometry. All aspects of patient care are addressed within the journal as well as the practice of optometry including economic and business analyses. Basic and clinical research papers are published that cover

Submit your manuscript here: https://www.dovepress.com/clinical-optometry-journal

\section{Dovepress}

all aspects of optics, refraction and its application to the theory and practice of optometry. The manuscript management system is completely online and includes a very quick and fair peer-review system, which is all easy to use. Visit http://www.dovepress.com/ testimonials.php to read real quotes from published authors. 